\title{
Tracking Structural Modifications from In-Situ Atom Probe Gas-Solid Reactions through Computational Homology
}

\author{
Scott Broderick ${ }^{1}$, Tianmu Zhang ${ }^{1}$ and Krishna Rajan ${ }^{1}$ \\ 1. Department of Materials Design and Innovation, University at Buffalo, Buffalo, NY, USA
}

We have developed an in-situ reaction chamber for atom probe tomography (APT) to experimentally measure in-situ gas-surface reactions as a function of time, temperature, and pressure [1]. We are able to look at a very broad range of gases and reactions and the ability to perform these measurements allows us to accurately perform calculations for a wider range of environments and providing a wider range of values for calibrating atom probe reconstructions and subsequent electronic structure modeling [2]. Therefore, combining this in situ atomistic measurement capability for surface reactions with computational protocols allows us to computationally address the issues related with surface degradation under extreme environments.

With the use of the in-situ chamber (Figure 1), we can measure the changes at the surface in terms of neighborhood chemistry and relative bond strengths. These surface-chemistry relationships have been compared and subsequently calibrated with electronic structure calculations to assess the degradation mechanisms [3-4]. This approach allows to identify the mechanism for degradation in terms of charge distribution, model the relative bond strengths, and measure the evaporation field (ie. the propensity to degrade) as a function of applied field, surface chemistry, and surface structure. When coupled with experimental measurements of the ingress of gases (Figure 2), a full understanding of the connection between degradation, surface reactivity, microstructural effects, and diffusion is developed. Further, this can be linked with ion evaporation maps to define how the atoms evaporate from the surface, whether as a single ion or as a bonded species. The statistics associated with the evaporation maps can be tracked back to the surface bonding, where atoms more likely to evaporate as a bonded species have stronger relative bond strengths. These computational platforms allow for enhanced understanding of the role of gas and material chemistry on the degradation of a material under extreme conditions.

However, the issue we explore here is that the assessment of crystal structure and the corresponding structural effects are not easily described in this process. For this reason, persistence diagrams will be developed that describe the structure through packing density and relative spatial positioning, with the specific 3D homological features defined. In this way, persistence diagrams are defined and correlated with relative atom sizes and nearest neighbor environments. By tracking these changes over an entire APT experiment, we can define the relative changes in the structure, describe the data systematics, and integrate a structural measurement component into the modeling framework previously described. This therefore expands our understanding of degradation and surface-gas reactions to chemistry-processinglocal structure relationships [5].

\section{References:}

[1] S. Dumpala, et al, Ultramicroscopy 141 (2014) p. 16.

[2] S. Srinivasan, et al, Ultramicroscopy 159 (2015) p. 381.

[3] J. Peralta, S. Broderick, K. Rajan, Ultramicroscopy 132 (2013) p. 143. 
[4] C. Loyola et al, Journal of Vacuum Science and Technology A 34 (2016) p. 061404.

[5] We acknowledge support from the National Science Foundation (NSF) under grant number DMR1307811.
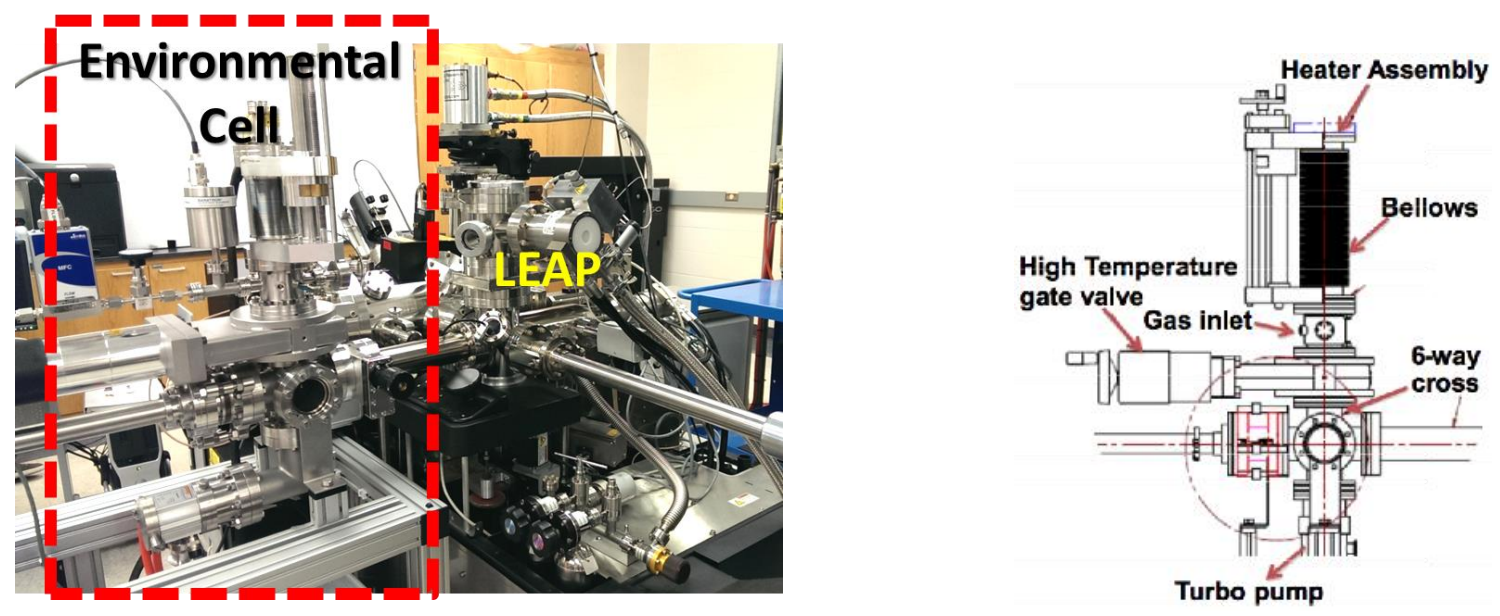

Figure 1. (a) Environmental cell integrated onto the atom probe for performing in-situ gas-solid reactions, as a function of temperature, pressure, and gas chemistry. (b) Schematic of the environmental cell, including the gas introduction system and the sample transfer mechanism (Adapted from [1]).
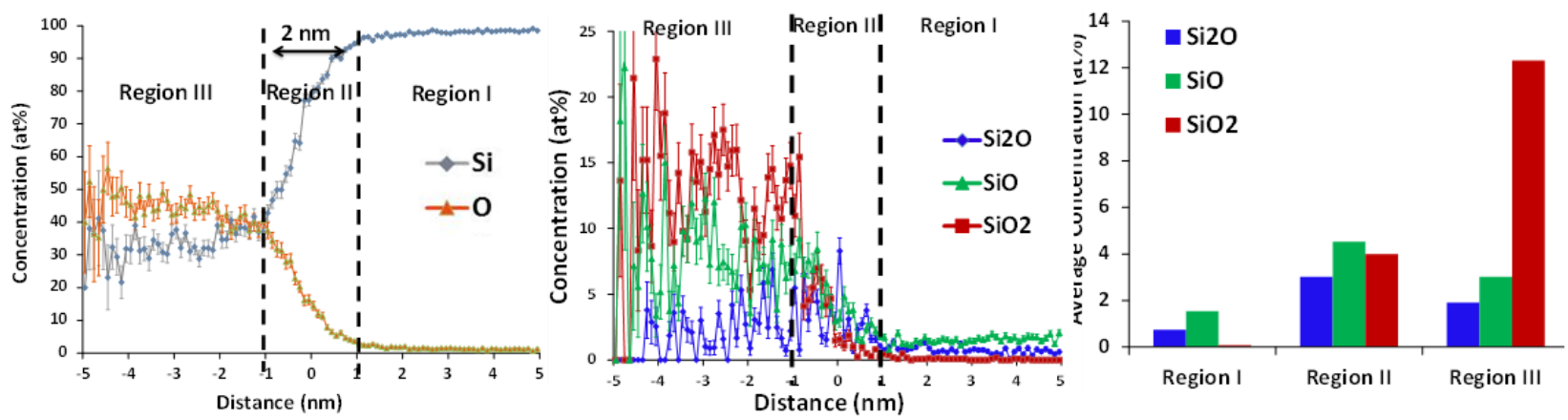

Figure 2. Results of in-situ reaction of Si. Comparison of the different gas ingress depths, layers of regions formed, suboxide ratios, and interfacial widths provides an added level of understanding to gassurface reactions. These measurements will be further analyzed through a computational homology framework, where the role of structure and atomic arrangement will be quantified and assessed. 